\title{
The Standard Model as a Philosophical Challenge
}

\author{
Edward MacKinnon*
}

\begin{abstract}
There are two opposing traditions in contemporary quantum field theory. Mainstream Lagrangian QFT led to and supports the standard model of particle interactions. Algebraic QFT seeks to provide a rigorous consistent mathematical foundation for field theory, but cannot accommodate the local gauge interactions of the standard model. Interested philosophers face a choice. The can accept algebraic QFT on the grounds of mathematical consistency and general accord with the semantic conception of theory interpretation. This suggests a rejection of particle ontology. Or they can accept the standard model on the grounds of its established success. This alternative, which I defend, suggests revising philosophical accounts of scientific theory and finding some way of accommodating particles.
\end{abstract}

*emackinnon@comcast.net 


\section{Introduction}

The standard model of particle physics is, arguably, the most successful fundamental model in the history of physics. It is theoretically capable of handling all the strong, electromagnetic, and weak interactions of quarks, leptons, gluons, W and Z bosons, and photons. Yet, it is conspicuously absent from many recent philosophical accounts of quantum field theory $(\mathrm{QFT})^{1}$ In these accounts the prevailing contention is that relativistic QFT does not support, and is not even compatible with, particle ontology. One way to understand the gap between physical accounts and philosophical reconstructions is to focus on the strategy choices facing philosophers. I will schematize this in terms of two interrelated sets of choices, one philosophical and the other physical.

The philosophical choice concerns theories about scientific theories. The familiar distinction between syntactic accounts, based on axiomatic formulations, and semantic accounts, based on the interpretation of a formalism through models, draws attention away from a shared presupposition. A necessary condition, presupposed by both methods is that the mathematical formulation of a theory should have a basic consistency independent of any physical interpretation. This is a requirement proper to an interpretative reconstruction of theories. In the normal practice of physics very few functioning theories meet this requirement.

There are two major traditions in quantum field theory. The mainstream tradition, which I will call Lagrangian quantum field theory (LQFT), but which most physicists simply call quantum field theory, is based on Lagrangians constructed by a variety of methods. Historically, the development began by quantizing classical, or pseudo-classical, Lagrangians. 
This was soon supplemented by the method of functional integration associated with Feynman diagrams, and other methods of quantization. The resulting quantum formulations were often modified to accommodate experimental results, expanded to include theoretical assumptions, and revised to yield desirable consequences. This is the general approach that led to the standard model of particle physics. Neither LQFT, nor the standard model, has a mathematical formalism that is consistent independent of the intended interpretations. For the present discussion I will grant that the standard model does not fit, and probably cannot be revised to fit, the basic norms of the semantic conception of theories. From this perspective LQFT and the standard model are not theories, but ugly collections of rules.

The opposing tradition is labeled 'algebraic', of 'axiomatic', or 'local' quantum field theory. On the assumption that the algebraic formulation is more general and that with plausible assumptions it can accommodate axiomatic quantum field theory (Haag 1992, sect. III) we will take algebraic quantum field theory (AQFT) as the alternative. AQFT pays a high price for mathematical rigor. It treats single particle states, interactions by perturbation theory and collisions by S-matrix theory. It does not include the particle interactions basic to the standard model. ${ }^{2}$

This division puts the basic choices facing concerned philosophers into stark relief. They can stick to a priori conceptions of what a scientific theory should be and judge the acceptability of QFT formulations by these norms. AQFT passes; the standard model does not. Alternatively, they can accept LQFT and the standard model on the grounds of their established success and then revise philosophical conceptions of scientific theories and the modes of interpreting them. This alternative encounters a methodological problem. The 
physicists who developed the standard model have paid very little attention to AQFT and, for the most part, have either ignored or explicitly rejected philosophical accounts of theory interpretation. As a result the second alternative has not been made philosophically intelligible. This is the task of the present paper. For this reason we will focus on interpretative perspectives and refer to other sources for technical details.

There are two contrasting interpretative perspectives: one explicit; the other implicit in a functioning tradition. To relate them we begin with a distinction between a theory of interpretation and the interpretation of a theory. To polarize the contrast we associate a theory of interpretation with the philosophical overseer. The overseer has an a priori conception of what a proper theory should be, a mathematical formalism given physical significance by morphisms (syntactic mode) or models (semantic mode). The contrasting figure is the field worker who advances field theory by any stratagem that looks promising The field worker typically regards mathematical formulations as tools, not foundations, ignores AQFT, and rejects any role for philosophical overseers. Yet, as the hermeneuticists insist, there is no reading of a text, following of a tradition, or advancement of a practice without an interpretation. Accordingly, the presentation of an alternative perspective involves an attempt to make some functional features of the implicit interpretation explicit. To make this philosophically intelligible we need a semantic ascent to an interpretative level. This will not compel assent, but should remove the obstacles blocking an informed choice of alternative strategies. 


\section{The AQFT Perspective}

The functional interpretation of a theory is reflected in the way a theory is reconstructed, whether for interpretative or pedagogical purposes. We will use this as a basis for comparing key features of both developments: methodology of development; interrelation of basic concepts, theory interpretation, and interpretative problems. Since our task is one of making the second perspective philosophically intelligible we will simply indicate the aspects of the AQFT interpretation that challenge the second perspective. Both begin by quantizing classical fields functionally represented by classical oscillators. AQFT now relies on Weyl canonical commutation relations. This exponentiates the $\hat{p}$ and $\hat{q}$ of the Heisenberg commutators, on the grounds that both cannot be jointly defined over all of Hilbert space. Since the Stone-von Neumann theorem does not apply to an infinite dimensional Hilbert space there is a problem of relating inequivalent representations. ${ }^{3}$ Quantization of classical oscillators with positive frequencies leads to commutation (or anti-commutation) relations for creation, $a^{\dagger}(k)$, and annihilation $a(k)$ operators. Repeated use of these operators leads from the vacuum, the lowest energy state of the system, to an n-particle state in Fock space and a number operator, $N=\int d^{3} a^{\dagger}(k) a(k)$ This is one component of the theoretical term 'particle' (or particle $_{t}$ ) as it functions in QFT. The group-theory component, stemming from Wigner, asks what kind of entity corresponds to an irreducible representation of the Poincaré group. It is an object characterized by mass, spin, parity, and various charges. A one-particle state in the corresponding Fock space is produced by a creation operator operating on the vacuum, $a^{\dagger}(k)=|k\rangle$. Particles $s_{t}$ are generated by repeated operation on the vacuum. The theory does not provide localization for particle $\mathrm{s}_{\mathrm{s}}$. 
A more promising approach towards a clarification of the theoretical concept, 'particle' is to see where this fits into a network of basic concepts: We will focus on the differing interrelations of five basic concepts: 'observable', 'field', 'state', 'particle', and 'local gauge'. A basic assumption of current AQFT is that local observables carry all the relevant physical information. There are, accordingly, two foundational presuppositions: Minkowski spacetime as a classical manifold $(\mathcal{M})$ with the familiar metric, $g$, and observables defined as functions of space-time. For a bounded space-time region, $\mathcal{O}$ one can construct an algebra of observables: $\mathcal{O} \rightarrow \mathcal{A}(\mathcal{O})$. This is a $W^{*}$-algebra closed with respect to weak limits and closed under the adjoint operation. (See Haag, Sect. III). This has the property of isotony,

$$
\mathcal{O}_{1} \subset \mathcal{O}_{2} \Rightarrow \mathcal{A}\left(\mathcal{O}_{1}\right) \subset \mathcal{A}\left(\mathcal{O}_{2}\right),
$$

leading to a net of algebras. Since different theories select different observables, an indefinitely large number of algebras is possible. The quasi-local algebra is the smallest $\mathrm{C}^{*}$-algebra containing all the local algebras. Two qualifications should be noted. At this general level one needs only space-time localization of observables, not particular values. Also, Einstein causality enters through the assumption that operator representing observables of space-like separated regions commute.

In the earlier development of QFT by Dirac and Schwinger, and in many contemporary developments, fields are assigned point values and a foundational role. AQFT treats observables, rather than fields, as basic. The classical analysis of field measurements by Bohr and Rosenfeld showed that field measurements only yield values spread out over the volume of a classical test particle. Symbolically, if $f$ is a smooth test function, then $\phi(f)=\int \phi(x) f(x) d^{4} x$. This yields operator-valued distributions, rather than a precise 
assignment of field values to particular points. States can be defined as positive, normalized linear functionals on the algebra of observables. The Gelfand-Naimark-Segal construction leads from *-algebra, $\mathcal{A}$, and algebraic states to a Hilbert space, $\mathcal{H}$, and a representation of $\mathcal{A}$ by linear operators acting in $\mathcal{H}$. In standard formulations of quantum physics, Hilbert-space states are the sources of non-locality problems, such as entanglement. The relation between algebraic and Hilbert-space states hinges on the representation of an algebra of observables. I will only consider one aspect of the complex topic, how particle localization figures in this theoretical framework.

Relativistic particle mechanics does not support the non-relativistic position operator, $<x>=\int \bar{\psi} \hat{x} \psi d^{3} x$. The equivalent relativistic position operator is not covariant and does not produce a position average. The effective position operator resulting from the NewtonWigner, or Foldy-Wouthuysen, reduction of the Dirac wave function is a non-relativistic operator that smears position over a volume about the size of the particle's Compton wavelength. The primacy accorded observables suggests localizing a particle by localizing its observables. Naively, a particle in a state is localized in a region, $O$ if all of its observables, $\hat{\mathcal{F}}$ have values, $\langle\psi|\hat{\mathcal{F}}| \psi\rangle=0$ for any region spacelike separated from $\mathcal{O}$. This, however, leads to incompatible eigenvalues assignments. One way around this is to specify differences relative to the vacuum. In Knight localization a system specified by a state, $\psi$, is localized in a region, $\mathcal{O}$, iff $\langle\psi|\hat{\mathcal{F}}| \psi\rangle-\langle\Omega|\hat{\mathcal{F}}| \Omega\rangle=0$ for any system observable localized in a region spacelike separated from $\mathcal{O}$. According to the Reeh-Schlieder theorem, however, the set of vectors, $\mathcal{F} \mid \Omega>$ obtained by applying the operator, $\hat{\mathcal{F}}$ to the vacuum state is dense in the Hilbert space of state vectors. It follows that relatively localized states span the Hilbert 
space, blurring any notion of sharp localization. ${ }^{4}$

Finally, there is the issue of the interactions specified by local gauge invariance. According to Haag's theorem (Haag, pp, 55-57, Earman and Fraser 2006) the canonical quantization process that leads to free fields cannot yield any unitarily equivalent interacting fields. The objects on which local gauge symmetry acts cannot be accommodated by the algebra of observables. So AQFT proponents tend to interpret away the distinctive physical significance the standard model accords local gauges. ${ }^{5}$ An opposed reading is possible. Since the standard model successfully treats local interactions, some of the presuppositions of Haag's theorem must be inapplicable.

From the AQFT perspective there are three principal difficulties with the standard model and its particle interpretation. The theory does not have a consistent or justifiable mathematical formulation. Relativistic quantum field theory does not support any coherent notion of localized particles. The postulated local gauge interactions cannot be included in a systematic development of QFT. There is a final difficulty that does not depend on an AQFT perspective. Even it staunchest supporters regard the standard model as a relatively lowenergy approximation to a deeper theory, which may not even be a field theory (Weinberg 1995, xxi). A phenomenological theory does not supply a basis for determining a fundamental ontology. So, the particle talk of the standard model should be interpreted as just a way of talking that relates theoretical predictions to the language of experimental physics, and not as an account of the basic furniture of the physical world. 


\section{The LQFT Perspective}

Basic texts on LQFT and the standard model, e.g. Weinberg, Kaku, Zee, Kane, Ticiatti, never treat the algebra of observables, the Stone-von Neumann theorem, inequivalent representations, Haag's theorem, the Reeh-Schlieder theorem, or theoretical problems of particle localization (except for condensed matter physics). One may handle this by repeating the opposed jokes about sloppy mathematics and physical contributions less than any pre-assigned epsilon. Or one may try to understand why these are not seen as pertinent issues. The latter alternative requires a radically different interpretative perspective, which I will attempt to indicate.

Philosophical accounts of theory interpretation often presuppose a formal system whose consistency is established independent of any physical interpretation accorded it. While this may fit theories as reconstructed, it rarely fits functioning theories, especially constructive theories. As Weinberg noted, ${ }^{6}$ the development of the standard model induced a revised understanding of how quantum field theory functions. The standard model emerged from a collective effort and merged components with their own proper constraints and modes of interpretation. I will list some features of the standard model that differ from widely accepted philosophical conceptions of theories. The principal ingredients were: quantum physics; special relativity; group theory, especially Lie groups and Lie algebras; an extension of symmetry principles to internal symmetries; the extension of gauge invariance from global to local gauges; and spontaneous symmetry breaking. The constraints included considerations of analyticity, unitarity, covariance, and fitting Feynman diagrams. There was a sustained collective effort to fuse these into a viable synthesis. The standard model is a collection of 
disjoint parts weakly unified by a particle ontology. Mathematical propriety was relegated to a mopping up operation.

Neither LQFT nor the standard model has a basic mathematical consistency independent of a physical interpretation. ${ }^{7}$ The struggle was to achieve a basic consistency of the standard model as a functioning physical theory, not an abstract mathematical formalism. This struggle focused on removing, or at least taming, the major sources of inconsistency. The electroweak component of the standard model had two basic sources of inconsistency. The first, already familiar from QED, involved a reliance on series expansions that have divergent terms and may not converge even when the divergences are covered over. This was originally handled by regularization and renormalization and eventually by the renormalization group. ${ }^{8}$ Because of anomalies related to chiral symmetry breaking, the electroweak component in not renormalizable when treated in isolation. It is renormalizable in an overall model including quarks. (See Kaku 1993, 414-429.) The second source of inconsistency involved the introduction of intermediate vector bosons. Introducing massive bosons violated covariance requirements; while massless bosons did not accord with short-range forces. The accepted solution involved introducing massless bosons and then invoking the Higgs mechanism to supply the needed mass. This leads to singular vacua that lack the translation invariance required by the Haag theorem.

Strong interactions presented different consistency problems. Because of the strength of the strong coupling constant one could not rely on series expansions. This was resolved through the discovery of quark confinement and asymptotic freedom. A reliance on the flavor quantum number as the basis for exchange forces led to inconsistencies with the Pauli 
exclusion principle. This was resolved by the introduction of a new color quantum number. The net result of these resolutions was a contextual consistency of the standard model as a theory of local gauge interactions. The contextual aspect is clearest when the standard model is regarded as an effective theory valid only within a limited energy range.

The semantic conception of theories relies on an ontological interpretation of theories. In the realist (anti-realist) mode it asks Feynman's forbidden question: What must (might) the world be like if a theory is true of it? Interpretation of a physical theory is seen as a model-mediated relation between a mathematical formalism and reality. Language, as such, plays no role in the interpretation. Interpretations of AQFT tend to shift the emphasis to the epistemological side of the epistemological/ontological circle, a move that favors an anti-realist stance. Haag, the patriarch of AQFT, was influenced by Bohr's stress on epistemological issues (Haag 2000) Others, notably Clifton Bub and Halvorson, were influenced by arguments assigning quantum information theory a foundational role in the interpretation of quantum mechanics. (see Clifton, Bub, and Halvorson 2003.) Here too the role of language is slighted. Observables, not reportables, supply the foundation for interpretation.

LQFT and the standard model rely on interpretation through a detailed correspondence with experimental results as reported. Two aspects of this should be noted. The first is an implicit reliance on language as an essential part of the process of interpreting the theory. Participants in the ongoing dialog rarely analyze the language used. A philosophical critic should consider the necessary conditions for unambiguous communication of physical information. The second is the changed relation between experimentation and theory consequent upon the emergence of the standard model, something emphasized by Picker- 
ing (1984). Prior to the development of the standard model experimenters discovered new particles $\left(\tau, \theta, q_{\text {strange }}, q_{\text {bottom }}, J / \phi\right)$, which theoreticians then attempted to explain. In the later, and now dominant stage, theoreticians predicted particles $\left(\Omega^{-}, W^{ \pm}, Z, q_{\text {charmed }}, q_{\text {top }}\right)$ and guided experimenters on the paths to observe the predicted particles.

To illustrate the significance of this shift we will consider one experiment that played a pivotal role both in establishing the standard model and also in the epistemological switch to theoretical considerations as the basis for guiding and interpreting experiments. After Gell-Mann predicted the existence of the $\Omega^{-}$particle he discussed experimental tests with Nicholas Samios, who directed high energy particle physics experiments at Brookhaven National Laboratory. Samios had access to an 80 inch bubble chamber and could produce a stream of high energy $K^{-}$mesons. The predicted path (omitting some details) from collisions of $K^{-}$particles with protons to $\Omega^{-}$detections was (Samios 1997)

$$
\begin{aligned}
K^{-} p & \rightarrow \Omega^{-} K^{+}\left(K^{0}\right) \\
\Omega^{-} & \rightarrow \Xi^{0} \pi^{-} \\
\Xi^{0} & \rightarrow \Lambda^{0} \pi^{0} \\
\pi^{0} & \rightarrow \gamma\left(\rightarrow e^{+} e^{-}\right)+\gamma\left(\rightarrow e^{+} e^{-}\right) \\
\Lambda^{0} & \rightarrow p \pi^{-}
\end{aligned}
$$

Surprisingly, the concept 'particle' in this experimental context is unproblematic. It is the classical concept of a small object with a precise location (or particle $_{c}$ ). This is not an ontological claim that this is what the $\Omega^{-}$or $e^{+}$really is. It is Bohr's complementarity principle specifying the experimental contexts in which the use of the classical concept is appropriate. This concept functions as part of the language used in the experimentaltheoretical discourse. Here language plays an implicit but important role in supporting the informal inferences needed for experimental analysis. Thus the classical concept 'particle' entails 'traveling in a precise trajectory', 'colliding with other particles', etc. This plays a 
role in tracking the $e^{+}$and $e^{-}$tracks back to their sources. It plays a crucial role in identifying the $\Omega^{-}$. If a $\Xi^{-}$had been produced there would be a straight-line trajectory between its production point (the origin of the $K^{-}$track) and the vertex of the $p-\pi^{-}$tracks. If an $\Omega^{-}$is produced there is a slight displacement due to the intermediate steps indicated above. (Samios 1997, 533). The analysts, including Staten Island housewives, had to keep examining successive frames until the deviation was found. The $\Omega^{-}$was finally detected on the frame numbered 97,025. AQFT accommodates experimental results by fitting events, such as clicks of a detector, into a theoretical framework. It does not accommodate the role of particle language in experimental inferences and in experimental-theoretical dialog. This dialog framed and guided the advances in particle physics.

The status accorded local gauge interactions is the crucial difference between the philosophical overseers and AQFT on one side and the field workers on the other. We should consider its status and implications. Global gauge invariance fits into the method of beginning with classical physics and relying on canonical commutation relations for quantization. The argument that local gauge invariance should be valid wherever global gauge invariance is valid is a heuristic argument. Local gauge invariance has the status of a postulate, not an inference. As already noted, neither local gauges nor the interactions they treat can be localized in the framework of observables. What status should be accorded such postulates?

Here three papers Einstein published a century ago supply a helpful guide. Einstein's earliest papers, which he later dismissed as worthless, attempted to infer the reality of atoms from such phenomena as capillarity and the electrical potential of dissolved salts. His Brownian paper postulated molecules of a definite size and inferred testable consequences, 
an exponential height relation of a solute in a solution. Lorentz addressed the experimental challenges to electrodynamics by beginning with the standard theory and adding corrections of order $(v / c)$ (stellar aberration, the Hoek-Fizeau experiment), and of order $(v / c)^{2}$ (the Michelson-Morley experiment). Einstein postulated the invariance of the speed of light and the covariance of the laws of physics to deduce Lorentz's equations. The light-quantum hypothesis was not justified by Planck's law and was only weakly related to Wien's law through the volume dependence of entropy. It was a postulate that flatly contradicted the accepted interpretation of electromagnetic vibrations as continuous. The attempts to interpret the significance of these breakthroughs by fitting them into a methodological extension of established physics all proved abortive. The new theories must be interpreted on the basis of the novel postulates.

The postulate of local gauge invariant interactions goes beyond, and is incompatible with, the framework of free fields proper to AQFT. Haag's theorem is not relevant. It shows that local gauge interactions cannot be inferred from a free-field basis. It cannot block the postulation of such interactions as the foundation of a new theory. The Reeh-Schlieder theorem is doubly irrelevant. The use of this theorem to refute locality hinges on the status of the vacuum as a cyclic vector. Here 'vacuum' does not refer to some objective nothingness. It is the lowest invariant state of the Hamiltonian. Gauge theory has a vacuum that is infinitely different, since each is defined for an infinite number of points. The interactions postulated by local gauge theory are sharply localized.

The nature of this localization can be illustrated by the simplest application. Consider 
the local phase transformation,

$$
\psi(\mathbf{x}, t)^{\prime}=e^{-\imath \theta \mathbf{x}} \psi(\mathbf{x}, t)
$$

This is local in the sense that the value of $\theta(\mathbf{x})$ can be different for different positions. To relate this to non-relativistic quantum mechanics as well as electrodynamics, use the Schrödinger wave equation,

$$
-1 / 2 m \nabla^{2} \psi(\mathbf{x}, t)=\imath \partial \psi(\mathbf{x}, t) / \partial t
$$

If in eq. (2) we substitute $\psi(\mathbf{x}, t)^{\prime}$ then we get

$$
-1 / 2 m\left[\nabla^{2}(-\imath \nabla \theta] \psi e^{-\imath \theta(\mathbf{x}, t)}\right]=\imath[\partial / \partial t-\imath \partial \theta(\mathbf{x}, t) / \partial t] e^{-\imath \theta(\mathbf{x}} .
$$

This definitely does not have the same form as eq (2). However, we may supplement eq. (1) with the local electromagnetic gauge transformation,

$$
\mathbf{A}^{\prime}=\mathbf{A}+1 / q \nabla \theta(\mathbf{x}), \quad V^{\prime}=V-1 / q \partial \theta(\mathbf{x}) / \partial t .
$$

For a particle of charge, $q$, the conjugate momentum is $\mathbf{p} \rightarrow \mathbf{p}-q \mathbf{A}(\mathbf{x})$. The standard quantization procedure is to replace $\mathbf{p}$ by $-\imath \nabla$. So,

$$
-\imath \nabla \rightarrow-\imath[\nabla-\imath q \mathbf{A}(\mathbf{x})] \equiv-\imath \mathbf{D}
$$

$\mathrm{D}$ is the covariant derivative. When this is substituted for the ordinary derivative and the two sets of phase changes are introduced, then the transformed Schrödinger equation has the same form as the untransformed equation. The covariant derivative used in the standard model can be written in symbolic form as:

$$
\partial / \partial x_{\mu} \rightarrow D / D x_{\mu}=\partial / \partial x_{\mu}-\imath g / \hbar L_{k} A_{\mu}{ }^{k}, \quad \text { where }
$$


$L_{k}$ are the generators of the gauge group, the $A_{\mu}{ }^{k}$ are the gauge fields, and $g$ is the matching coupling constant. This covers all basic interactions: hardron-hardron, hardron-lepton, and lepton-lepton, and fit all empirical results until the recent (2002) discovery that neutrinos have mass (not allowed by the standard model). All couplings are effectively represented by covariant derivatives. This is a renormalizable theory. These couplings are simply postulated and then the coupling constants are adjusted to fit the empirical data.

The use of the same spatial point, $(\mathbf{x})$, for both the wave equation and the electromagnetic potential provides a sharp localization for the interaction. The assumption is that this sharp localization obtains as a matter of fact. However, the localization is not related to the framework of observables that plays a basic role in the application of the Reeh-Schlieder theorem. Since the localization is not specified relative to an observer's system, there is no foundation for addressing the covariant properties of the (non)-specification. In this context it is appropriate to speak about the interaction of sharply localized particles. Many defenders of AQFT would grant this, but argue that this way of speaking does not support an ontology of particles. The problem here is the significance accorded 'ontology'. One might, my preference, use the rather minimal sense proper to analytic philosophy, a clarification of the basic commitments implicit in the language used. Or, one might opt for a stronger sense, going beyond what physics says to delineate the basic furniture of reality. This calls for a separate study. Here I will only make one point. Feynman's forbidden question is forbidden, in accord with Bohrian semantics, because any proposed answer goes beyond the limits of what can be meaningfully said. One may disagree with this restriction, but cannot treat the issue without a serious consideration of the role of language in the interpretation of physics, 
something systematically omitted from the semantic conception.

The standard model systematizes particle interactions. Intrinsic to the theory are three families of leptons and quarks, the photon, three intermediate vector bosons, and eight gluons. Other particles, especially Higgs particles, axions, and magnetic monopoles, are assumptions or tentative consequences of the model. The final difficulty concerns the status of these particles when the standard model is interpreted as a low energy approximation to a not yet developed deeper theory. This should not be seen as a project of theory replacement. At issue here is the status of effective theories and their commitments. The general principle is that a theory can be regarded as an effective theory up to a proper high-energy cutoff. Above that level new physics is needed. The new physics supplements, but does not replace, the old physics. Interaction terms in an effective theory are divided into two classes: non-renormalizable terms, which are negligibly small; and renormalizable terms. These depend on the value of the cutoff only through the values of parameters. These are set by experimental results, not a fundamental theory. Thus, the development of the standard model did not change atomic physics, or its commitment to atoms. The energy gap between the standard model and string theory is about ten orders of magnitude greater than that between atomic physics and the standard model. Hopefully, a new depth theory will explain the parameters now fed by hand into the standard model. It will not change the standard model. The commitment to quarks and leptons should remain a permanent part of physics. Even if they are eventually explained as something like different modes of vibration of a string or membrane, they still function as distinct particles at the energy level proper to the standard model. Strict reductionists might discount such relative ontology. The are 
ontological commitments of the dialog of physics, not a depiction of the basic furniture of reality as it exists independent of human knowledge. I believe that this is all that can be meaningfully said on the issue. However, considerations of meaning and language are beyond the scope of the present paper. 


\section{Notes}

${ }^{1}$ See, e. g., Halvorson and Clifton 2002, Halvorson 2001, Ruetsche 2002, Redhead and Wagner 1998.

${ }^{2}$ Appraisals of the present state of AQFT are given in Haag 1992, Buchholz 1998, Buchholz and Haag 1999, and Halvorson and Muger 2006

${ }^{3}$ This is treated in Halvorson and Clifton 2000 and in Ruetsche 2002.

${ }^{4}$ The problems of particle localization are treated in Wallace 2001, Halvorson and Clifton 2001, and Ruetsche 2002.

${ }^{5}$ Thus, Haag 1992, 299 claimed that incorporation of the local gauge principle is the central challenge facing AQFT. When it was not incorporated he interpreted it as transformations of unobservable fields. Buchholz 1998, 2 claims: "From the more fundamental [i.e., AQFT] point of view the gauge field appears to be nothing but a device for the construction of the local (gauge invariant) observables of the theory in some faithful representation, usually the vacuum representation." To implement the goal of deriving the physics of the standard model from a rigorous mathematical basis the Clay mathematical institute is offering a million dollar prize for solving the problem: Prove that for any compact simple gauge group, $G$, quantum Yang-Mills theory on $\Re^{4}$ exists and has a mass gap $\Delta>0$. See http://www.claymath.org/millenium.

${ }^{6}$ Weinberg 1997. The historical development is presented in Riordan 1987, Hoddeson et 
al 1997, and in Cao 1998. Appraisals of its functioning as a theory are given in Wilczek 1998, 1999, 2002.

${ }^{7}$ The mathematical formulation of standard LQFT is given a detailed exposition in Ticciati 1999.

${ }^{8}$ A philosophical appraisal of interpretations of renormalization may be found in Teller 1995, chap. 7. The special problems encountered in renormalizing gauge theories are summarized in the articles by Veltman and 't Hooft in Hoddeson et al 1997. 


\section{References}

Buchholz, Detlev. (2000), "Algebraic Quantum Field Theory: A Status Report",arXiv:math$\mathrm{ph} / 0011044 \mathrm{v} 1$.

Buchholz, Detlev and Rudolf Haag (1999), "The Quest for Understanding in Relativistic Quantum Physics", arXiv:hep-th/9910243.

Cao, Tian Y. (1998). Conceptual Developments of 20th Century field Theories. Cambridge: Cambridge University Press.

Clifton, Rob, Jeffrey Bub, and Hans Halvorson, (2003), "Characterizing Quantum Theory in terms of Information-Theoretic Constraints," Foundations of Physics 33: 1561-1591.

Clifton, Rob and Halvorson, Hans (2001), "Are Rindler Quanta Real? Inequivalent Particle Concepts in Quantum Field Theory", philsci-archive.pitt.edu/archive/00000073.

Dosch, Hans, Guenter Mueller, F. Volkhard,and Norman Sieroka (2002), "Quantum Field Theory: its Concepts Viewed from a Semiotic Perspective", philsci-rchive.pitt.edu/archive/0000073.

Earman, John and Doreen Fraser. (2006), "Haag's Theorem and its Implications for the Foundations of Quantum Field Theory", PhilSciArchives 2673.

Haag, Rudolf. (1992). Local Quantum Physics: Fields, Particles, Algebras. Berlin: SpringerVerlag.

Haag, Rudolf (2002), "Questions in quantum physics: a personal view", arXiv:hep-th/0004006.

Halvorson, Hans (2001), "Reeh-Schlieder Defeats Newton-Wigner," Philosophy of Science 68: $111-133$.

— and Rob Clifton (2002), "No Place for Particles in Relativistic Quantum Theories", Philos- 
ophy of Science 69: 1-28.

— and Michael Muger. (2006). "Algebraic Quantum Field Theory", PhilSciArchives 2633.

Hoddeson, Lillian, Laurie Brown, Michael Riordan, and Max Dresden (1997). The Rise of the Standard Model: Particle Physics in the 1960s and 1970s. Cambridge: Cambridge University Press.

Kaku, Michio (1993). Quantum Field Theory: A Modern Introduction. New York: Oxford University Press.

Kane, Gordon (1993). Modern Elementary Particle Physics. Reading, MA: Addison-Wesley Publishing Company.

Pickering, Andrew. (1984). Constructing Quarks: A Sociological History of Particle Physics. Chicago: University of Chicago Press.

Redhead, Michael L. G. and Fabian Wagner (1998). "Unified Treatment of EPR and Bell Arguments in Algebraic Quantum Field Theory", arXiv: quant-ph/9802010.

Ruetsche, Laura (2002), "Interpreting Quantum Field Theory," Philosophy of Science 69: 348378.

Samios, Nicholas (1997), "Early Baryon and Meson Spectroscopy Culminating in the Discovey of the Omega-Minus and Charmed Baryons", In Hoddeson and et al, 525-541.

't Hooft, Gerard (1997), "Renormalization of Gauge Theories", in Hoddeson et al, 145-178.

Teller, Paul (1995). An Interpretive Introduction to Quantum Field Theory. Princeton: Princeton University Press.

Ticciati, R. (1999). Quantum field Theory for Mathematicians. Cambridge: Cambridge University Press. 
Wallace, David (2001), "Emergence of particles from bosonic quantum field theory", arXiv:quant$\mathrm{ph} / 0112149 \mathrm{v} 1$.

--. (2001), "In Defence of naiveté: The conceptual status of Lagrangian quantum field theory", arXiv:quant-ph/0112148.

Weinberg, Steven (1995). The Quantum Theory of Fields. Vol. I : Foundations. Cambridge: Cambridge University Press.

—- (1997). "Changing Attitudes and the Standard Model", in Hoddeson et al., 36-44.

Wilczek, Frank (1998), "Quantum Field Theory", arXiv:hep-th/9803075.

- (1999), "What QCD Tells Us About Nature-and Why We Should Listen", arXiv:hepth/9907340.

--. (2002), "QCD and Natural Philosophy", arXiv:physics/0212025.

Zee, A (2003), Quantum Field Theory in a Nutshell. Princeton: Princeton University Press. 


\section{Interaction Energies}

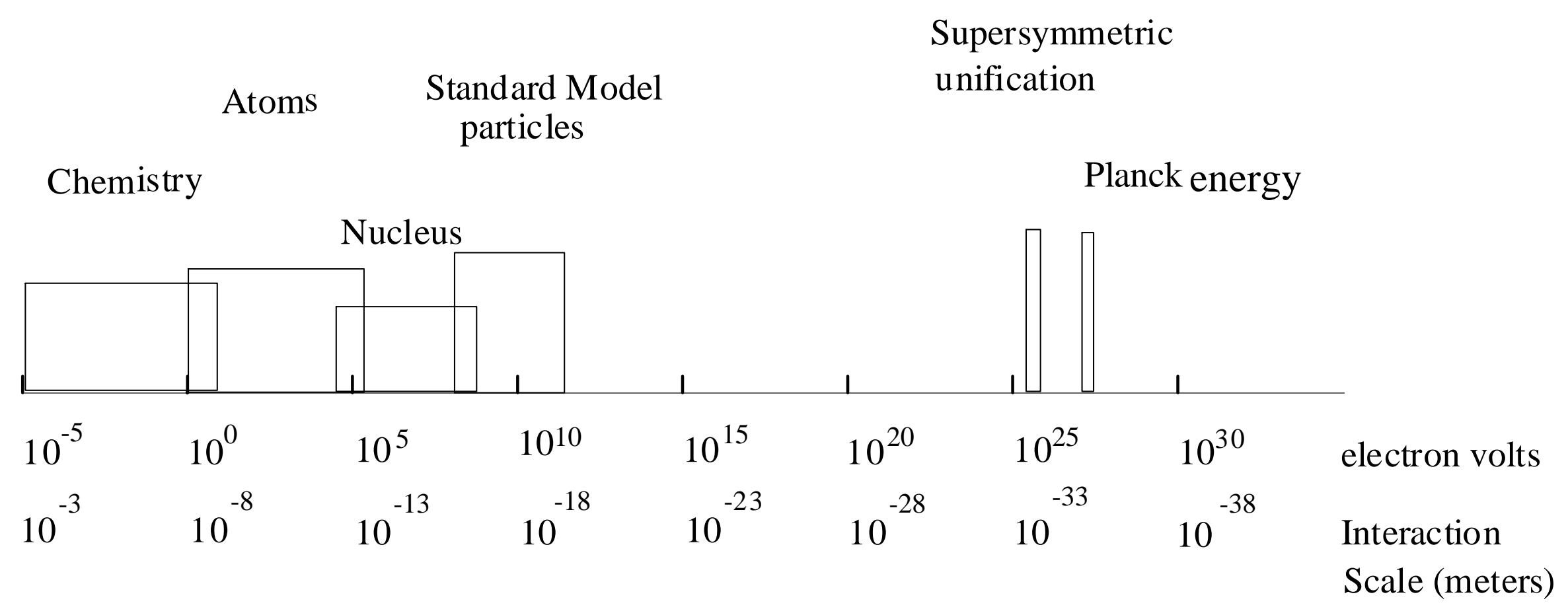

